\title{
A technique for section thickness evaluation for microphotometry and image analysis of sectioned nuclei
}

\author{
R.L. Cabrini, A. Folco, S. Orrea, M.T. Savino, A.M. Schwint and M.E. Itoiz* \\ Oral Pathology Department, Faculty of Dentistry, University of Buenos Aires, Argentina \\ Radiobiology Department, National Atomic Energy Commission, Argentina
}

Received 8 October 1997

Revised 1 October 1998

Accepted 15 October 1998

\begin{abstract}
The exact knowledge of the section thickness is a requisite for making the necessary corrections on DNA measurements in tissue sections. Several methods have been proposed to evaluate section thickness, each of them with advantages and disadvantages depending on the type of specimen and equipment available. We herein report another method based on preparation of standard material whose optical density varies as a function of its thickness and is sectioned and measured alongside the tissue specimen. The standards consist of celloidin cylinders stained with the PAS reaction and embedded in paraffin. For prior characterization of the cylinders, sections of different thickness were obtained and mounted. The optical density of each section was measured by direct microphotometry or image analysis. The actual thickness of each section was evaluated following re-embedding of piled groups of sections in a paraffin block and transversal sectioning. The thickness was then measured with a micrometric eye-piece. Optical density and actual thickness of each section were plotted on a normogram curve. Once a given tissue is sectioned alongside with the reference cylinder, the actual thickness is determined by its optical density on the normogram curve.
\end{abstract}

Keywords: Section thickness, DNA cytometry, image analysis

\section{Introduction}

DNA microphotometry on tissue sections, both by direct scanning microphotometry or by image analysis usually requires correction of the measured values to correct for errors derived from evaluation of nuclear fractions instead of whole nuclei $[1,6,7,12]$. These corrections are based on mathematical algorithms developed to calculate the whole content of DNA the area measured and the section thickness is known $[3,5,9,11]$.

Over or underestimation of the actual section thickness are important error sources when the algorithms are applied. Since measurements by interference microscopy or other precise methods $[2,4,10]$ are difficult to employ in routine DNA studies, most corrections directly use the value of the microtome setting, although the actual section thickness can differ greatly from the setting [1]. Moreover, this difference may be very variable depending on the nature of the material and the size and temperature of the specimen.

\footnotetext{
*Correspondence: Dr M.E. Itoiz, Anatomia Patologica, Facultad de Odontologia, M.T. de Alvear 2142, (1122) Buenos Aires, Argentina.
} 


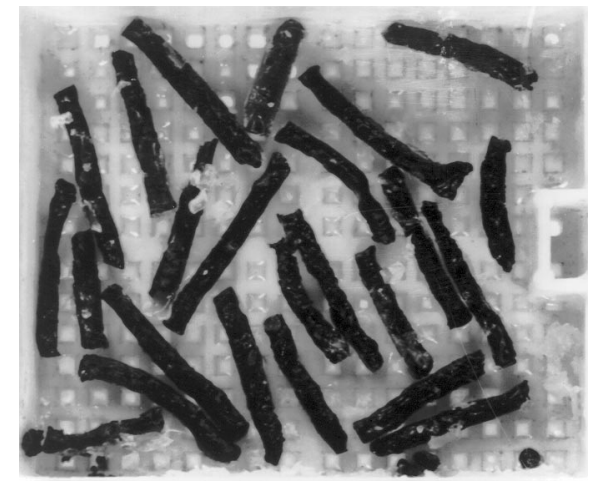

Fig. 1. Embedding cassette with celloidin cylinders after staining, dehydration and paraffin embedding.

Gschwendtner and Mairinger [8] developed a method to determine the actual section thickness of paraffin sections based on re-embedding of a part of the section to be resectioned orthogonally to the original thickness and measured with a standard calibrated eye piece. This method albeit accessible and reliable, adds considerable technical work to each case and is very difficult to apply to small specimens. To overcome these difficulties, we developed a method based on the preparation of standard material whose optical density varies only as function of its thickness.

\section{Material and methods}

Cylinders of about $3 \mathrm{~mm}$ in diameter and $1 \mathrm{~cm}$ in height were prepared with a biopsy needle from the following materials after gelification:

- agar $2 \%$ in distilled water;

- gelatin 5 to $10 \%$ in distilled water;

- celloidin 7\% in equal parts of absolute alcohol and ethylic ether, hardened in alcohol $70 \%$.

The whole cylinders were stained by the following methods:

- eosine $2 \%$

- ferric hematoxyline;

- PAS reaction: peryodic acid $1 \%$ overnight, washing in distilled water for $1 \mathrm{~h}$, Schiff solution in darkness and postfixation in $10 \%$ formaline for $6 \mathrm{~h}$;

- acid fucsine $1 \%$;

- Verhoeff's solution (for staining of elastic fibers).

All stains were tested from $1 \mathrm{~h}$ to overnight.

Stained cylinders were then washed $(2 \mathrm{~h}$ in running water), dehydrated over a $6 \mathrm{~h}$ period in graded alcohols, embedded in paraffin and sectioned by a conventional rotary microtome (American Optical Co.) set at $5 \mu \mathrm{m}$ (Fig. 1). Sections were placed on slides and processed employing the routine technique until mounting in balsam.

Microscopic examination revealed that celloidin cylinders stained with the PAS reaction as described above, employing the Schiff solution overnight yielded best results regarding their homogeneity, volumetric changes, suitability of staining and reproducibility (see results below). Thus, only the celloidinPAS cylinders were chosen to be submitted to the following procedure. 

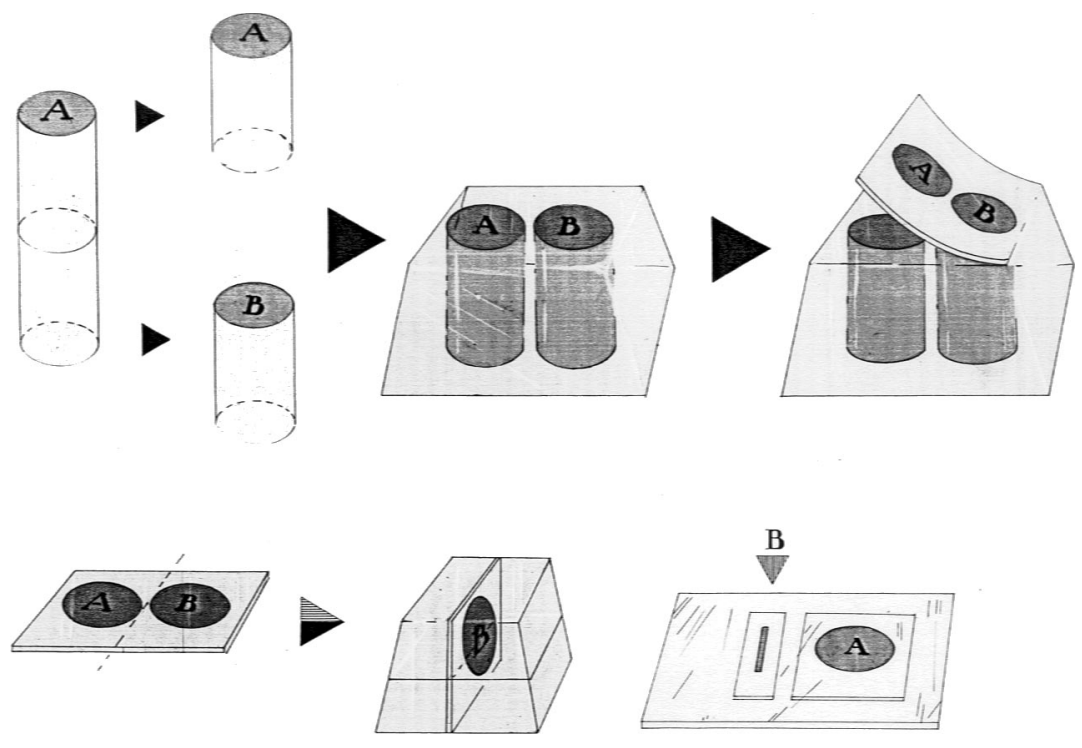

Fig. 2. Schematic representation of the steps followed to measure the optical density and the actual section thickness of a PAS-cylinder. (See text for explanation.) This procedure was repeated with sections of 3, 5, 7, 10, 15 and $20 \mu \mathrm{m}$ in thickness from the same block.

The cylinder was half sectioned and the two halves ( $\mathrm{A}$ and $\mathrm{B}$ ) were embedded in a single paraffin block which was then sectioned at different microtome settings: 3, 5, 7, 10, 15, and $20 \mu \mathrm{m}$. Each section was then cut with a razor blade to separate the cylinders. Sections from one cylinder (A) were mounted on a slide maintaining the order of the microtome settings. The corresponding sections of the other cylinder (B) were piled up and embedded in melted paraffin to make a single block which was then orthogonally sectioned through the thickness of the original sections (Fig. 2).

After dehydration and mounting the mean optical density of the A sections was measured by means of two different techniques:

- $X-Y$ scans of a $100 \times 100 \mu \mathrm{m}$ central area of the section using a MPM 80 Zeiss Cytoscan with a $2 \mu \mathrm{m}$ diameter light spot and the APAMOS program.

- Evaluation of a predetermined central area of about $100 \mu \mathrm{m}$ in diameter on the screen of a KONTRON Zeiss image analyser, using the IBAS-DNA program.

The actual thickness of each section was assessed from the B sections by means of a micrometric eye piece.

The complete procedure was repeated with 4 blocks of paired cylinders processed in 4 different staining batches.

Mean optical densities were then plotted against the thickness.

The following additional trials were performed:

- $X-Y$ scans of the entire cylinder sections, using the APAMOS program with a $2 \mu \mathrm{m}$ diameter spot, to test the homogeneity of the staining reaction within the cylinders.

- Mean optical density measurements of ten cylinders stained in the same batch and embedded and sectioned in a single block to demonstrate the homogeneity between cylinders to be used as standards. 


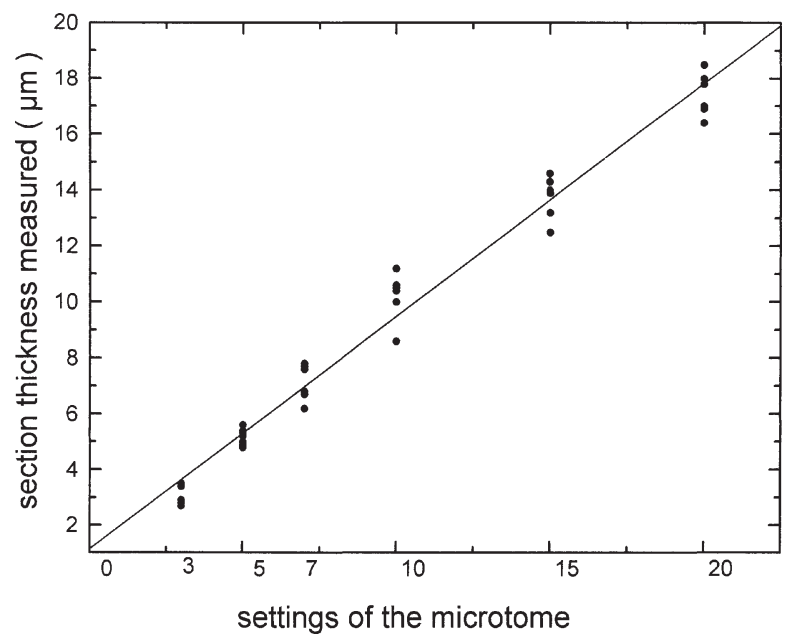

Fig. 3. Linear relationship between the settings of the microtome and the actual thickness of sections of a PAS stained cylinder.

\section{Results}

Gelatin and agar yielded cylinders which undergo irregular volume reduction during the embedding process. Conversely, celloidin cylinders were easily handled without size modifications.

Among the staining reactions tested, PAS gave a strong and homogeneous purple color. Eosine, ferric hematoxyline, acid fucsine and Verhoeff's solutions gave clearer areas in the central part of the cylinders due to slow penetration of staining solutions.

Figure 3 shows the relationship between the settings of the microtome and the actual section thickness. For the conditions and material used, the values were similar within the range of use.

Mean optical densities measured by direct microphotometry and image analysis varied within the range of 0.01 to 0.06 units of optical density.

The adjusted curve of mean optical densities of PAS-celloidin sections plotted against their actual thickness for a total of 50 to 60 measurements for each staining batch gave a parabolic curve due to saturation of the readings of optical density for thickness in excess of $10 \mu \mathrm{m}$. For thickness values between 3 to $10 \mu \mathrm{m}$, data can be fitted by a straight line for practical purposes, given that the linear correlation was $r=0.97, p<0.001$ (Fig. 4). Since thicknesses below and above this values are not useful for routine DNA determinations, the straight line can be used as a normogram for thickness determination when the optical density is measured on a standard cylinder sectioned from the same block as the tumour specimen.

Mean optical densities of the 10 different cylinders processed in a single batch were almost identical.

$X-Y$ scans made possible the observation of point by point density values through a whole cylinder section expressed in a colour-code diagram. Only a peripheric area $1 \mu \mathrm{m}$ wide exhibited lower values, possibly due to the influence of flare (Fig. 5).

\section{Discussion}

Since the proposed standard yielded a linear correlation within a useful range of thicknesses and no variations were observed between the cylinders of the same batch, a number of cylinders can be prepared 

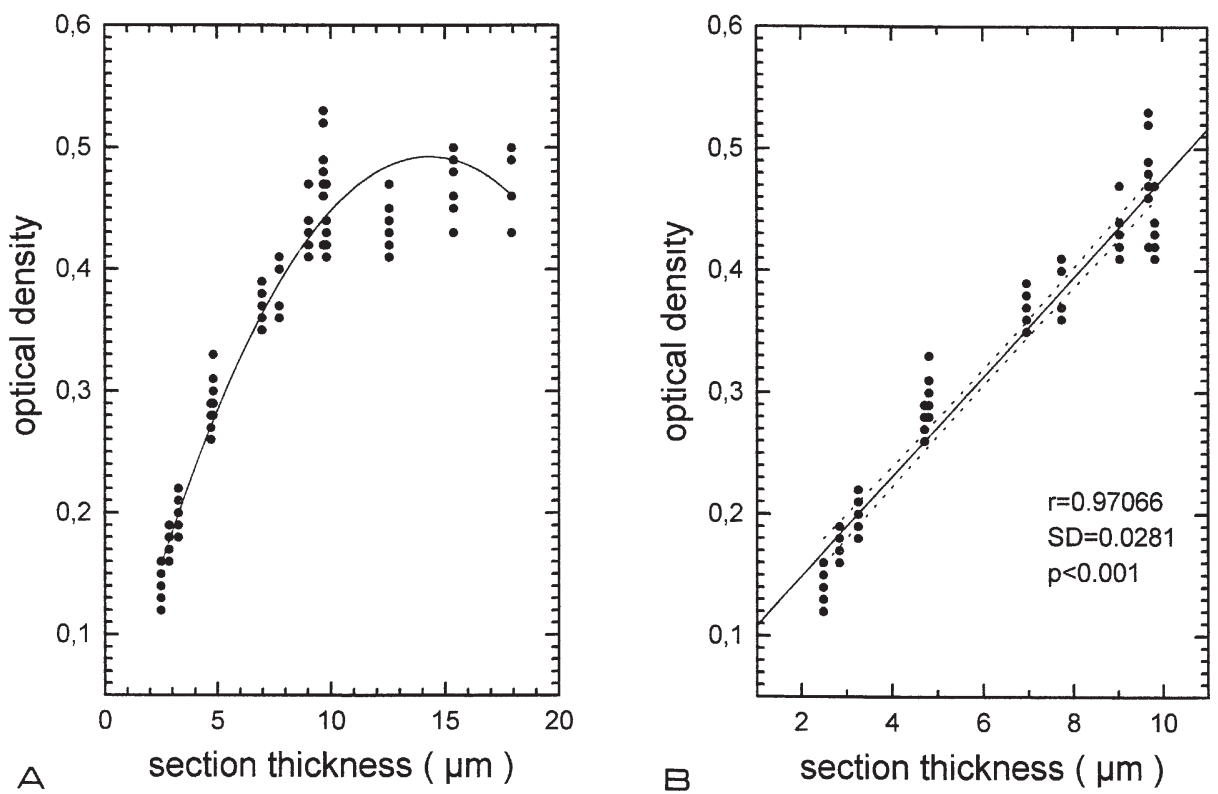

Fig. 4. (A) Optical densities plotted against actual thickness of sections taken from cylinders of the same staining batch, fitted by a parabolic curve by the "origin" software. Values beyond $10 \mu \mathrm{m}$ thickness deviate from linearity. (B) The same values as in (A), with exception of those greater than $10 \mu \mathrm{m}$, can be adjusted to a straight line. This line can be used, for practical purposes, as a normogram to determine thickness from optical density values of the cylinders used as standards.

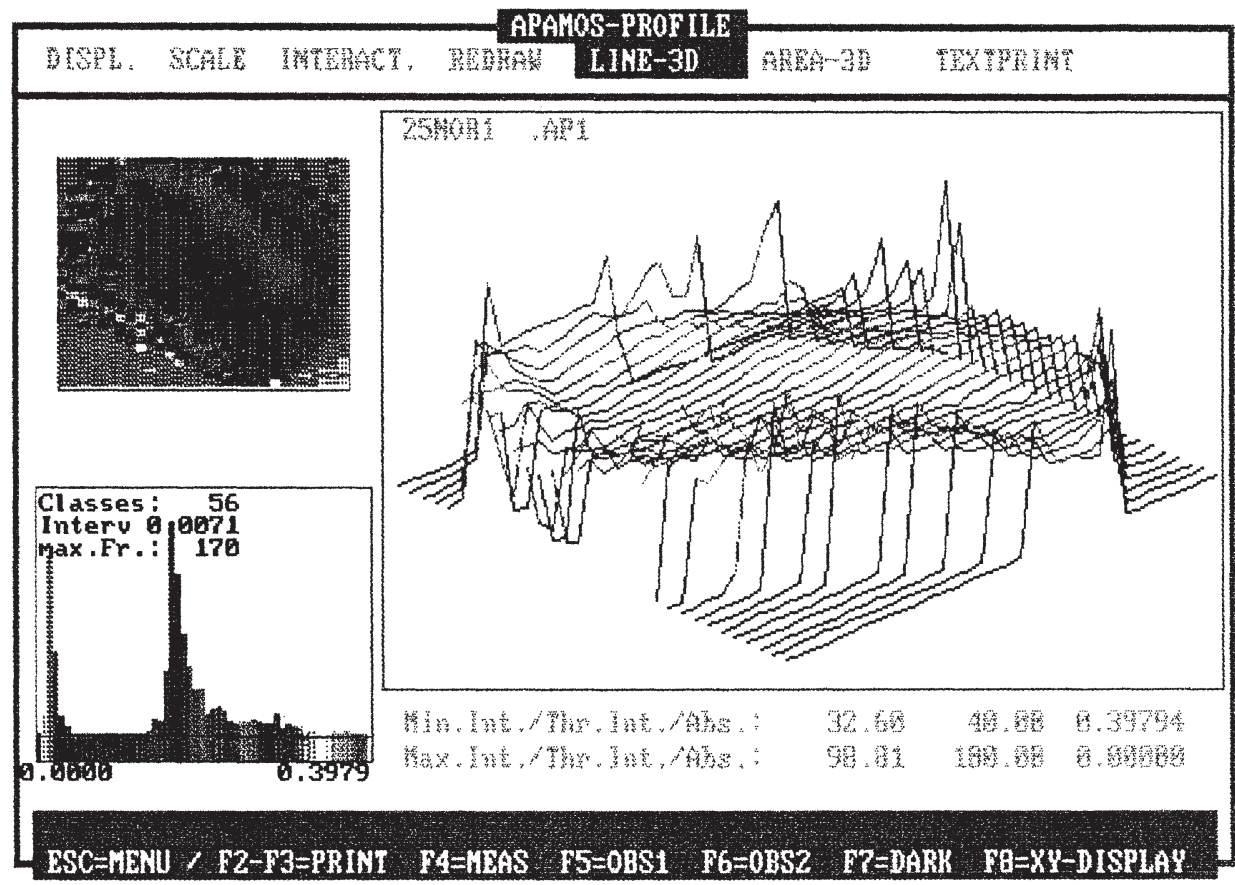

Fig. 5. Printout of the APAMOS program from an $X-Y$ scan of a cylinder section. Homogeneity of the stain in the central area of the section can be evaluated employing the optical density profile and a colour-code diagram. A narrow area in the periphery of the section exhibits higher values due to the flare effect. 
and stored in paraffin blocks. A very simple procedure is required to adhere one cylinder to a tumour block by slight melting of the paraffin. Once the cylinder and the tumour are simultaneously sectioned, the Feulgen reaction must be performed only on the tumour section, avoiding overstaining of the cylinder. This may be easily accomplished if the cylinder is mounted on one end of the slide by separating both specimen sections with a razor blade at the time of mounting. If the mean optical density of the cylinder is determined prior to evaluation of the tumour section, the thickness is given by the normogram curve.

The whole procedure of standards and normogram preparation must only be repeated when all the cylinders from a single batch have been used. Morover, each cylinder can be separated from the tumour block and reused for further determinations.

PAS-celloidin standards are easily prepared and sectioned. This fact explains the similarity between values obtained for the actual thickness and the microtome settings. However, in the case of specimens of different types or sizes, microtome settings can lead to marked errors in assigning a thickness value to a given section.

The proposed methodology may be particularly useful for DNA quantitation of large nuclei with diameters greater than the section thickness which actually require the application of correction formulae.

\section{Acknowledgements}

This work was supported by grants OD 007 from University of Buenos Aires and PMT-PICT 0161 from the CONICET (Argentine Research Council).

\section{References}

[1] R.T. Allison and J.F. Vincent, Measuring the forces acting during microtomy by the use of load cells, J. Micros. 159 (1990), 203-210

[2] A. Anthony, G.J. Colurso, T.M.A. Bocan and J.A. Doebler, Interferometric analysis of intrasection and intersection thickness variability associated with cryostat microtomy, Histochemical J. 16 (1984), 61-60.

[3] M. Bins and F. Takens, A method to estimate the DNA content of whole nuclei from measurements made on thin tissue sections, Cytometry 6 (1985), 234-237.

[4] K.A. Chaubel, Z. Ledin and J. Pilny, A new approach to the determination of thickness of the biological specimens by two wavelength method using interference microscopy, Acta Histochem. (1976), 131-143.

[5] G.D. Davies, Estimation of number and diameter of isodiametric spherical particles in microtome sections, J. Micros. 98 (1972), 79-83

[6] Y.S. Fu and T.L. Hall, DNA plody measurements in tissue sections, Anal. Quant. Cytol. 7 (1985), 90-96.

[7] A. Gschwendtner, A. Kreczy, K. Prein, C. Schonthaler and T. Mairinger, DNA cytometry: diploid standard and section thickness, Am. J. Clin. Pathol. 102 (1994), 558-559.

[8] A. Gschwendtner and T. Mairinger, How thick is your section? The influence of section tickness on DNA-cytometry on histological sections, Analytical Cell Pathol. 9 (1995), 29-37.

[9] T. Mairinger, A. Gschwendtner, G. Mikuz and V. Kempf, Tissue section image analysis. Comparison of different software releases, Am. J. Clin. Pathol. 101 (1994), 673.

[10] A.D. Pearse and R. Marks, Further studies on section thickness measurement, Histochemical J. 8 (1976), 383-386.

[11] J.P. Rigaut and A. Persoz, The "corpuscle" stereological problem. Re-evaluation using slab fragment volumes and application to the correction of DNA histograms from sections of spherical nuclei, J. Micros. 156 (1989), 371-382.

[12] Z. Sapi, J.B. Hendricks, P.G. Pharis and E.J. Wilkinson, Tissue section image anlysis of breast neoplasms, Anat. Pathol. 99 (1993), 714-720.

[13] H. Schimmelpenning, K. Hamper and U.G. Falkmer, Methodologic aspects of DNA assessment by means of image cytometry in tumours of the salivary glands. A comparison between the results obtained using sections and cytospin preparations from the same paraffin embedded specimens, Anal. Quant. Cytol. 11 (1989), 379-383. 


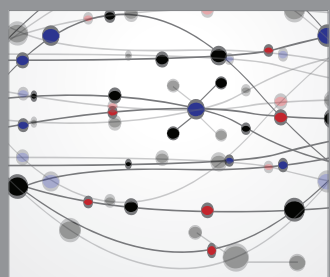

The Scientific World Journal
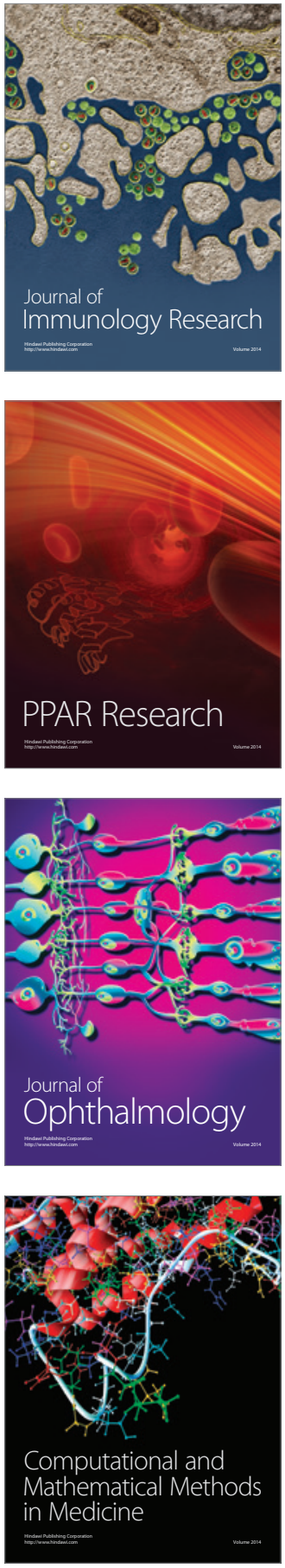

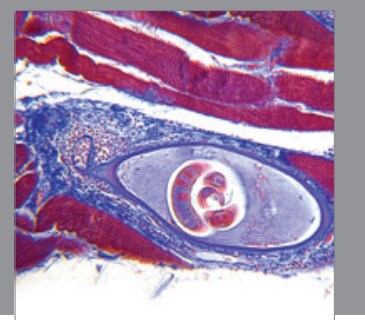

Gastroenterology

Research and Practice
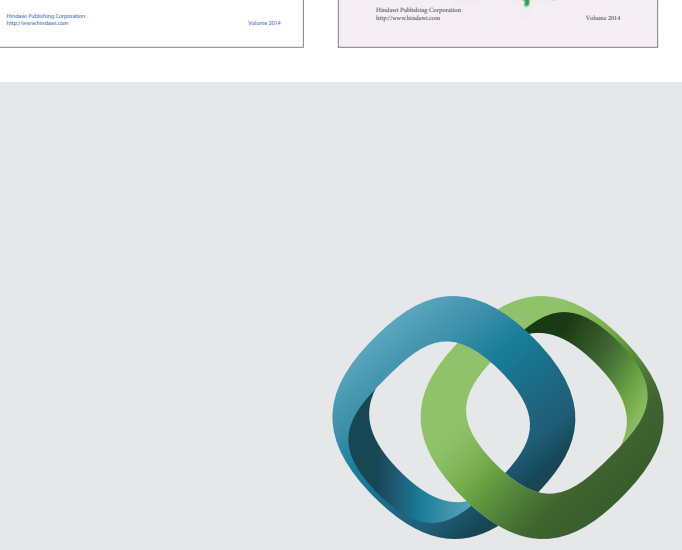

\section{Hindawi}

Submit your manuscripts at

http://www.hindawi.com
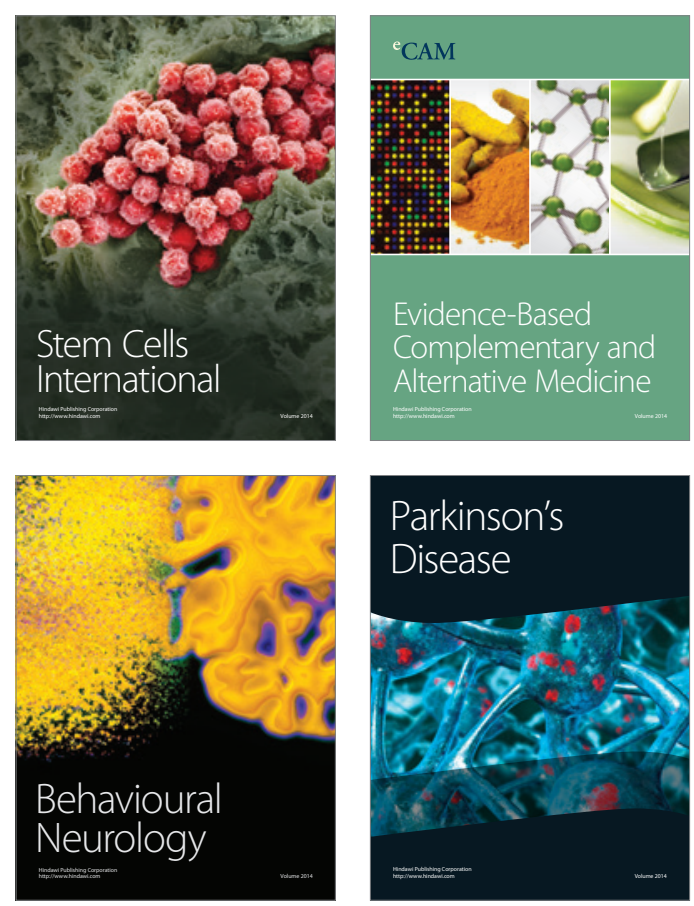

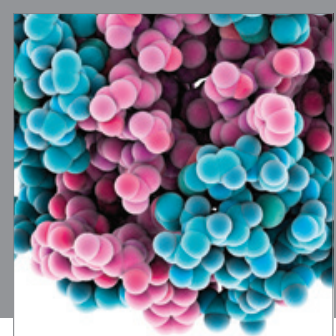

Journal of
Diabetes Research

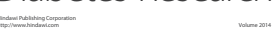

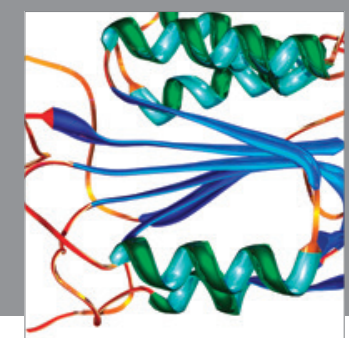

Disease Markers
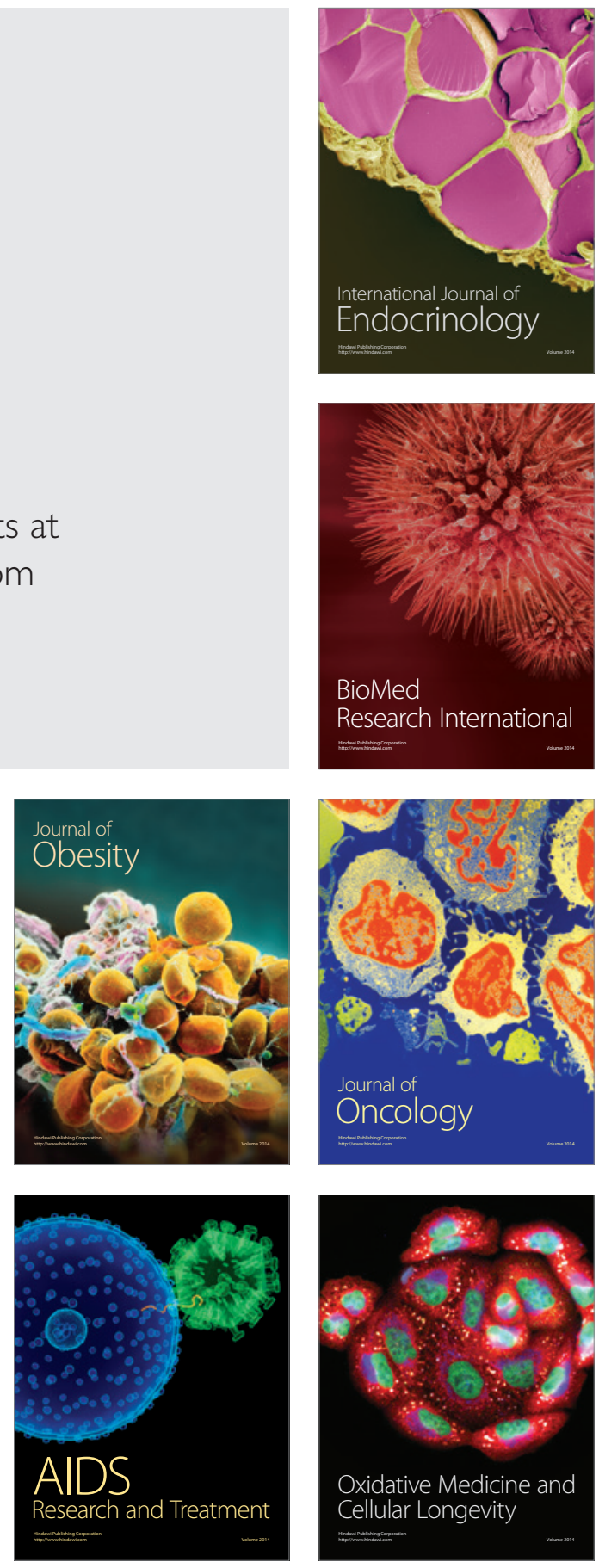